\title{
KIM-1 and NGAL: new markers of obstructive nephropathy
}

\author{
Anna Wasilewska • Katarzyna Taranta-Janusz • \\ Wojciech Dębek • Walentyna Zoch-Zwierz • \\ Elżbieta Kuroczycka-Saniutycz
}

Received: 6 December 2010 /Revised: 5 January 2011 /Accepted: 6 January 2011 /Published online: 31 January 2011

(C) The Author(s) 2011. This article is published with open access at Springerlink.com

\begin{abstract}
Congenital obstructive nephropathy is the primary cause of chronic renal failure in children. Rapid diagnosis and initiation of the treatment are vital to preserve function and/or to slow down renal injury. The aim of our study was to determine whether urinary (u) kidney injury molecule-1 (KIM-1) and neutrophil gelatinase-associated lipocalin (NGAL) may be useful non-invasive biomarkers in children with congenital hydronephrosis (HN) caused by ureteropelvic junction obstruction. The study cohort consisted of 20 children with severe $\mathrm{HN}$ who required surgery (median age 2.16 years) and two control groups (control group 1:20 patients with mild, non-obstructive $\mathrm{HN}$; control group 2: 25 healthy children). All of the children had normal renal function. Immunoenzymatic ELISA commercial kits were used to measure uKIM-1 and uNGAL concentrations. The preoperative median uKIM-1/creatinine (cr.) and UNGAL levels were significantly greater in the children with severe $\mathrm{HN}$ than in both control groups. Three months after surgery, UNGAL had decreased significantly $(p<0.05)$ in the children with severe $\mathrm{HN}$, but was still higher than that in control group 2 children $(p<0.05)$. Receiver operator characteristic analyses revealed a good diagnostic profile for uKIM-1 and UNGAL in terms of identifying a differential renal function of $<40 \%$ in $\mathrm{HN}$ patients (area under the curve (AUC) 0.8 and 0.814 , respectively) and $<45 \%$ in all examined children (AUC 0.779 and 0.868 , respectively). Based on these results, we suggest that increasing uNGAL and uKIM-1 levels are associated with
\end{abstract}

A. Wasilewska $(\triangle) \cdot K$. Taranta-Janusz $\cdot$ W. Dębek

W. Zoch-Zwierz $\cdot$ E. Kuroczycka-Saniutycz

Department of Pediatrics and Nephrology,

Medical University of Białystok,

Waszyngtona 17 ,

15-274 Białystok, Poland

e-mail: annwasil@interia.pl worsening obstruction. Further studies are required to confirm a potential application of UKIM-1 and UNGAL as useful biomarkers for the diagnosis and progression of chronic kidney disease.

Keywords Children · Kidney injury molecule-1 . Neutrophil gelatinase-associated lipocalin · Ureteropelvic junction obstruction $\cdot$ Chronic renal failure

\section{Introduction}

According to data of The North American Pediatric Renal Transplant Cooperative Study (NAPRTCS), obstructive nephropathy due to congenital or acquired urinary tract obstruction is the first primary cause of chronic renal failure (CRF) in children [1].

Congenital obstructive nephropathy results from maldevelopment of the urinary tract in the fetus, with the most common lesion being localized to the ureteropelvic junction (UPJ). The prevalence of this abnormality in children is 1 in 1500 of the general population [2]. The etiology of UPJ obstruction (UPJO) is multifactorial and polygenic, similar to most other anomalies of the urinary tract. Chronic urinary tract obstruction leads to progression of the renal injury, even after surgical management of the obstruction.

Currently available clinical tests, including renal ultrasonography, nuclide renal scans or plasma, and urine creatinine concentration are not good predictors of the future course of the disease, stressing the urgent need for new, simple markers of obstructive nephropathy that may be useful in the clinical assessment of the suitability of patients for surgical therapy and renoprotective intervention. Plasma or urine markers, including neutrophil gelatinase-associated lipocalin (NGAL), cystatin $\mathrm{C}$, and 
asymmetric dimethylarginine (ADMA), are recognized as the most promising potential biomarkers for the early detection and monitoring of acute kidney injury (AKI) and chronic kidney disease (CKD) [3]. More recently, the interest of clinicians has focused on the potential of kidney injury molecule-1 (KIM-1) as a biomarker. In this study we assessed the urine concentration of KIM-1 and NGAL molecules as markers of renal function in children with congenital hydronephrosis (HN).

NGAL (also known as lipocalin-2, siderocalin, uterocalin, and 24p3) belongs to the lipocalin family [4]. However, like many other endogenous biomarker molecules, it is not produced by one cell type. This ubiquitous $25-\mathrm{kDa}$ protein is secreted by various types of human tissues, including the gastrointestinal tract, respiratory tract, and the kidneys. In the kidneys, NGAL is secreted into the urine by the thick ascending limb of loop of Henle and collecting ducts of the kidney, with synthesis in the distal nephron $[5,6]$. Because of its small molecular size, NGAL is freely filtered and can be easily detected in urine. Urinary NGAL (uNGAL) is a very early and sensitive biomarker of kidney injury, and various studies have reported that uNGAL levels are markedly increased in AKI, diabetic nephropathy, nephritic syndrome, tubulointerstitial nephritis, and immunoglobulin A (IgA) nephropathy (IgAN) [7-9].

KIM-1 is a type I transmembrane glycoprotein with an ecto-domain containing both an Ig-like and a mucin domain. It was discovered in renal tubular epithelial cells in a screen for molecules involved in the pathogenesis of AKI [10]. In healthy kidneys, KIM-1 is undetectable. However, the increased expression of this protein was found at very high levels on the apical membrane of proximal tubule cells after ischemic and nephrotoxic injury $[10,11]$. KIM-1 expression is absent in the glomerulus, peritubular interstitial cells, or inner medullary cells $[10$, 12]. Another characteristic of KIM-1 that make it an ideal biomarker for kidney injury is the rapid and integrated cleavage of its ecto-domain into the lumens of kidneys tubules, which can make it detectable in urine [13]. The uKIM-1 level has been documented to be closely related to tissue KIM-1 level and to correlate with kidney tissue damage [14]. Clinical studies have documented that KIM-1 is upregulated in tubules of patients with focal glomerulosclerosis, IgA nephropathy, or membranoproliferative glomerulonephritis [15] and that it is associated with proteinuria and its consequences, such as tubular injury and interstitial fibrosis [15]. These results have led to the suggestion that uKIM-1 may be a promising, non-invasive biomarker of chronic tubulointerstitial damage.

The aim of the case-control study reported here was to evaluate uKIM-1 and uNGAL levels in young children and adolescents affected by severe congenital $\mathrm{HN}$ caused by UPJO. We also assessed the possible clinical application of
uKIM-1 and uNGAL as non-invasive diagnostic and predictive biomarkers in UPJO.

\section{Patients and methods}

\section{Patients}

This was a case-control prospective study performed in children with severe congenital HN caused by UPJO, who were diagnosed at the Department of Pediatrics and Nephrology and treated at the Pediatric Surgery Department of the Medical University of Białystok, Poland. The study was performed from January 2008 to February 2010. Healthy children were chosen from those referred to a pediatric outpatient clinic of the Children's University Hospital, Białystok, Poland, where all children are periodically monitored for their development and growth. All caregivers of the children were interviewed and gave informed consent for the children to participate in the study.

The children were categorized into three groups: one study group and two types of control groups. The study group included 20 children (15 boys, 5 girls; median age 2.16 years, range $0.16-17$ years) with severe $\mathrm{HN}$ due to unilateral, critical degree ureteral stenosis, who underwent pyeloplasty by the Anderson-Hynes method. The first control group (control 1) included 20 children with mild, non-obstructive $\mathrm{HN}$ who did not require surgery (median age 8.75 years); these children had suspected but not confirmed UPJO. The second control group (control 2) consisted of 25 healthy children (14 boys, 11 girls; median age 5.0 years, range $0.33-16$ years). All the children had a normal ultrasound examination of the kidney.

Health status was determined on the basis of the subjects' medical history, parental report, and routine laboratory examinations to rule out the presence of acute or chronic disease. The UPJO was confirmed with radionuclide renal scans. Criteria for pyeloplasty included positive findings on the clinical, ultrasound, and radionuclide scan evaluation. Renal ultrasound (US) was used as an initial diagnostic tool to detect HN. All US examinations were performed by a pediatric radiologist using a SSA280A scanner (Toshiba, Tokyo, Japan) equipped with a 3.5-MHz probe. The degree of $\mathrm{HN}$ was graded according to the Society for Fetal Urology (SFU) classification: grade 1 represents a split pelvis; grade 2 is further dilation of the renal pelvis, with a few visualized calyces permissible; grade 3 is renal pelvis dilation, with many distended calyces; grade 4 is a grade 3 appearance with the addition of thinned parenchyma [16]. Vesicoureteral reflux was ruled out in all patients by voiding cystourethrography (VCUG). The diagnosis of non-obstructive HN was made when there was (1) no change in differential renal function 
and the clearance half-life $\left(\mathrm{t}_{1 / 2}\right)$ on the follow-up technetium$99 \mathrm{~m}$ mercaptoacetyltriglycine (99mTc-MAG3) diuretic renography and (2) stationary HN on the US scan.

Criteria for inclusion in the study group were: (1) an age of 1 month to 18 years; (2) unilateral pelvicaliceal system (PCS) dilatation; (3) a 99mTc-MAG3 diuretic renogram (furosemide washout test) that suggested unilateral UPJ obstruction (study group) and differential renal function (DRF) available. A DRF of the affected dilated kidney of $\geq 45 \%$ was considered normal and a DRF of $<45 \%$ was considered abnormal. Exclusion criteria were associated anomalies, including vesicoureteral reflux, ureterovesical junction obstruction and posterior urethral valves obstruction, bilateral $\mathrm{HN}$, previous operation on the urinary system and other deformations of the external genital organs, deformations in the lower part of the ureter, bladder, and urethra, urinary stones, neurogenic bladder dysfunction, and "supranormal function" of the affected kidney (DRF $>55 \%$ ).

In the study group the urine samples were collected three times: the first morning voided urine samples obtained preoperatively (exam A); urine samples from affected pelvis obtained during the surgery (exam B); first morning voided urine samples collected 3 months after surgery (exam C). The clinical work-up included the analysis of medical charts to determine age, gender, laterality, and grade of hydronephrosis, anteroposterior (AP) pelvic diameter, age at diagnosis, method of treatment, measurement of height, weight, blood pressure, and physical examination. The biochemical work-up included the determination of serum creatinine concentration (measured by the Jaffe reaction), urea, glomerular filtration rate $\left(\mathrm{ml} / \mathrm{min} / 1.73 \mathrm{~m}^{2}\right)$, estimated by the Schwartz formula (eGFR): GFR $=\mathrm{k} \times \mathrm{G}(\mathrm{cm}) / \mathrm{Lcr}(\mathrm{mg} / \mathrm{dl})$, where $\mathrm{k}$ is the age-dependent coefficient $(0.55$ in boys $<12$ years old and girls at any age, 0.7 in boys $>12$ years of age), $G$ is growth, and Lcr is the level of creatinine in serum. The urine was aseptically collected between 7 and 8 am from the morning sample. Measurements of uKIM-1 and uNGAL were performed in samples frozen at $-80^{\circ} \mathrm{C}$. Patients with pathological leukocyturia were excluded from the examination.

\section{Methods}

KIM-1 ELISA assay uKIM-1 was determined using a commercially available enzyme-linked immunosorbent assay (ELISA) test kit (USCN Life Science, Hankou, Wuhan, China) and was expressed in nanograms per milligram creatinine ( $\mathrm{ng} / \mathrm{mg}$ cr.). In brief, a biotinylated polyclonal antibody specific for KIM-1 was used to detect KIM-1 in the urine samples. Horseradish peroxidase-conjugated avidin was added, followed by a color-forming peroxidase substrate containing tetramethylbenzidine. The color was then measured at $450 \mathrm{~nm}$ by a microtiter plate reader and compared with a standard curve. KIM-1 levels below the detection level were scored as $0.07 \mathrm{ng} / \mathrm{ml}$.

NGAL ELISA assay uNGAL was measured using a commercially available ELISA kit (BioPorto Diagnostics, Gentofte, Denmark) according to manufacturer instructions. The enzymatic reaction was quantified in an automatic microplate photometer. The urinary creatinine concentration was used to normalize NGAL measurements to account for the influence of urinary dilution on its concentration. uNGAL levels were expressed as uNGAL/cr. ratio in nanograms per milligram creatinine ( $\mathrm{ng} / \mathrm{mg} \mathrm{cr}$.). The mean intra- and inter-assay coefficients of variation for NGAL were 3.6 and $7.9 \%$, respectively. The detection limit was a mean of $0.012 \mathrm{ng} / \mathrm{ml}$.

\section{Statistics}

Data analysis was performed using the computer program Statistica ver. 9.0 (SPSS, Chicago, IL). Nonparametric statistics was chosen as the patient population of this study was relatively small. Statistical analysis was performed using the nonparametric Mann-Whitney $U$ test. Differences between the treatments were analyzed by Friedman's analysis of variance (ANOVA) for repeated measures. The receiver operating characteristic (ROC) curve was used to determine the cut-off values of uKIM-1 and uNGAL that gave the best sensitivity and specificity. Correlations between UNGAL and other variables were evaluated by the Pearson or Spearman test as appropriate.

\section{Ethics statement}

The study was approved by the ethics committee of the Medical University of Białystok in accordance with the Declaration of Helsinki. Written informed consent was obtained from all parents following an explanation of the purpose of the study.

\section{Results}

The demographic and clinical data for each group are summarized in Table 1. In our study cohort, boys were more frequently affected with $\mathrm{HN}$ than girls; this gender bias has also been reported in the literature [17]. The left kidney was more commonly involved than the right kidney (26:14, respectively). Grade $1 \mathrm{HN}$ was present in four children (10\%), and grades 2,3 , and $4 \mathrm{HN}$ were present in $11(27.5 \%), 14(35 \%)$, and $11(27.5 \%)$ children, respectively. Mean age at surgery was approximately 2 years. The DRF in the study group ranged from 10.2 to $45 \%$ (median 
Table 1 Summary of the clinical parameters of all studied patients
Values given in parenthesis are the range - unless stated otherwise

\begin{tabular}{|c|c|c|c|}
\hline \multirow[t]{2}{*}{ Clinical parameters } & Study group & Control 1 & Control 2 \\
\hline & \multicolumn{3}{|l|}{ Median (range) } \\
\hline Gender, $n$ (male/female) & $20(15 / 5)$ & $20(13 / 7)$ & $25(14 / 11)$ \\
\hline Age at diagnosis (years) & $0.29(0.06-15)$ & $4.5(0.08-17)$ & - \\
\hline $\begin{array}{l}\text { Age at the moment of examination } \\
\text { (years) }\end{array}$ & $2.16(0.16-17)$ & $8.75(0.75-17)$ & $5.0(0.33-16)$ \\
\hline $\begin{array}{l}\text { Clinical diagnosis (SFU grading) } \\
\text { Grade } 1\end{array}$ & - & 4 & - \\
\hline Grade 2 & - & 11 & \\
\hline Grade 3 & 9 & 5 & \\
\hline Grade 4 & 11 & - & \\
\hline Laterality (left/right) & $15 / 5$ & $11 / 9$ & - \\
\hline Differential renal function (\%) & $39(10.2-45)$ & $45.8(35-50)$ & - \\
\hline Length of affected renal pelvis (mm) & $38.5(21-70)$ & $31.0(16-41)$ & - \\
\hline Width of affected renal pelvis (mm) & $26.0(14.5-47)$ & $21.0(4-35)$ & - \\
\hline $\begin{array}{l}\text { Estimated glomerular filtration rate } \\
\left(\mathrm{ml} / \mathrm{min} / 1.73 \mathrm{~m}^{2}\right)\end{array}$ & $154.7(105.5-223.7)$ & $186.6(122.2-271.5)$ & $155.0(111.5-233.7)$ \\
\hline
\end{tabular}

$39 \%$ ) and was significantly lower than that in patients from control $1 \quad(p<0.05)$. The pelvis length was found to be significantly higher in study group than in control 1 children $(p<0.05)$. A radionuclide scan was not performed in healthy children, so the DRF was not assessed in this group.

Table 2 and Fig. 1 show that the preoperative uKIM- $1 / \mathrm{cr}$. levels from voided urine (exam A) and the affected pelvis (exam B) were significantly greater than those in both control groups: exam A, median 3.13 (range 0.6-10.73) ng/mg cr.; exam B, median 3.42 (range 0.19-9.59) ng/mg cr. versus control 1, median 1.04 (range $0.18-5.69, p<0.05$ ) ng/mg cr. and control 2, median $0.58(0.15-3.01 \mathrm{ng} / \mathrm{mg} \mathrm{cr}$., $p<0.01)$. Three months after surgery (exam $\mathrm{C}$ ), the uKIM in absolute values $(\mathrm{ng} / \mathrm{ml})$ did not differ from those of control 1; however, when these were corrected for creatinine, the difference was statistically significant.

The median uNGAL/cr. level was significantly higher in patients from the study group when compared to those of control 2. The highest levels of uNGAL/cr. were found in urine obtained from the affected pelvis during surgery. The difference between the uNGAL/cr. level from voided urine (median 23.66, range $2.04-60.35 \mathrm{ng} / \mathrm{mg} \mathrm{cr}$.) and the affected pelvis (median: 38.48 , range $1.5-131.23 \mathrm{ng} / \mathrm{mg}$ cr.) was not statistically significant $(p>0.05)$. Three months after surgery, the uNGAL/cr. level had decreased significantly $(p<0.05)$, and did not differ from control 1 patients $(p>0.05)$, although it was still higher than that of control 2 patients $(p<0.05)$. Similarly, the $\mathrm{uNGAL} / \mathrm{cr}$. level was significantly higher in control 1 patients than in the healthy control 2 children $(p<0.05)$.

To investigate the effect of pyeloplasty on uKIM-1/cr. and $\mathrm{uNGAL/cr}$. levels, we analyzed the values before and after surgery in specific patients. As shown in Fig. 2, after surgery the uKIM/cr. and uNGAL/cr. levels decreased significantly in 75 and $90 \%$ of patients, respectively.

A negative correlation between the $\mathrm{KIM}-1 / \mathrm{cr}$. ratio and DRF was found in the study group $(r=-0.312, p<0.01)$.

Table 2 Urine concentration of KIM-1 and NGAL in patients with UPJO (study group and control 1) and healthy controls (control 2).

\begin{tabular}{|c|c|c|c|c|}
\hline Exam ${ }^{\mathrm{a}} /$ group & uKIM-1 (ng/ml) & uKIM/cr. (ng/mg cr.) & uNGAL (ng/ml) & uNGAL/cr. (ng/mg cr.) \\
\hline Exam A (voided urine before pyeloplasty) & $1.35^{\mathrm{A}, \mathrm{b}}(0.96-6.76)$ & $3.13^{\mathrm{A}, \mathrm{b}}(0.6-10.73)$ & $8.38^{\mathrm{A}, \mathrm{b}}(3.3-55.22)$ & $23.66^{\mathrm{A}, \mathrm{b}}(2.04-60.35)$ \\
\hline $\begin{array}{l}\text { Exam B (urine samples from affected } \\
\text { pelvis during surgery) }\end{array}$ & $1.37^{\mathrm{b}}(0.27-5.3)$ & $3.42^{\text {B, b }}(0.19-9.59)$ & $12.43^{\mathrm{A}, \mathrm{b}}(1.78-100.0)$ & $38.48^{\mathrm{B}, \mathrm{b}}(1.5-131.23)$ \\
\hline $\begin{array}{l}\text { Exam } C \text { (voided urine } 3 \text { months } \\
\text { after surgery) }\end{array}$ & $1.41^{\mathrm{b}}(0.6-4.88)$ & $1.73^{\mathrm{A}, \mathrm{b}}(0.48-6.94)$ & $3.37^{\mathrm{b}}(1.09-55.3)$ & $5.62^{\mathrm{a}}(2.15-25.78)$ \\
\hline $\begin{array}{l}\text { Control } 1 \text { (children had suspected } \\
\text { but not confirmed UPJO) }\end{array}$ & $0.84^{\mathrm{b}}(0.23-2.71)$ & $1.04(0.18-5.69)$ & $3.94^{\mathrm{b}}(1.23-12.15)$ & $3.8^{\mathrm{a}}(0.9-44.77)$ \\
\hline Control 2 (healthy children) & $0.37(0.15-1.82)$ & $0.58(0.15-3.01)$ & $1.64(0.25-5.77)$ & $2.31(0.27-16.27)$ \\
\hline
\end{tabular}

uKIM-1, Urinary kidney injury molecule-1; uNGAL, urinary neutrophil gelatinase-associated lipocalin; cr., creatinine; UPJO, ureteropelvic junction obstruction

Values are given as the median with the range given in parenthesis

$p^{\mathrm{A}}<0.05$ to control $1, p^{\mathrm{a}}<0.05$ to control $2 ; p^{\mathrm{B}}<0.01$ to control $1, p^{\mathrm{b}}<0.01$ to control 2 
a)

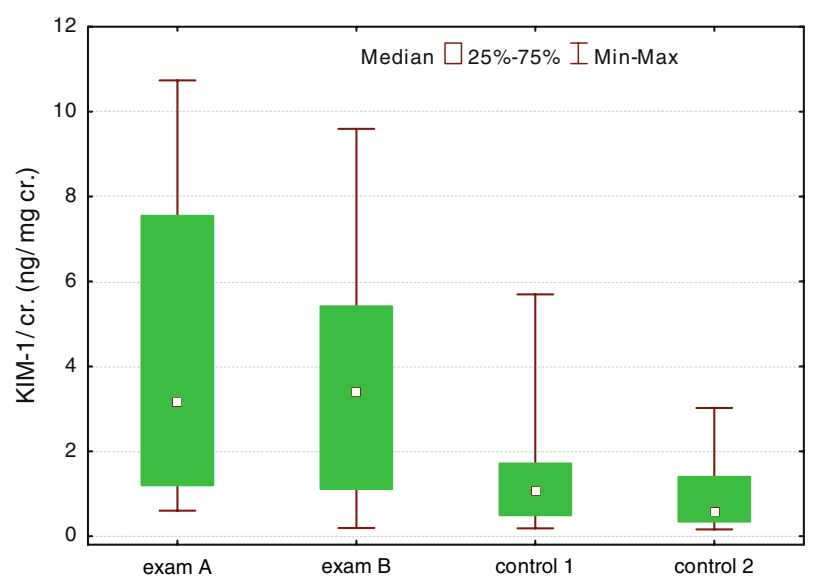

ANOVA test: Chi quadrat $=8.828236 \mathrm{df}=3 \mathrm{p}=.0317$

b)

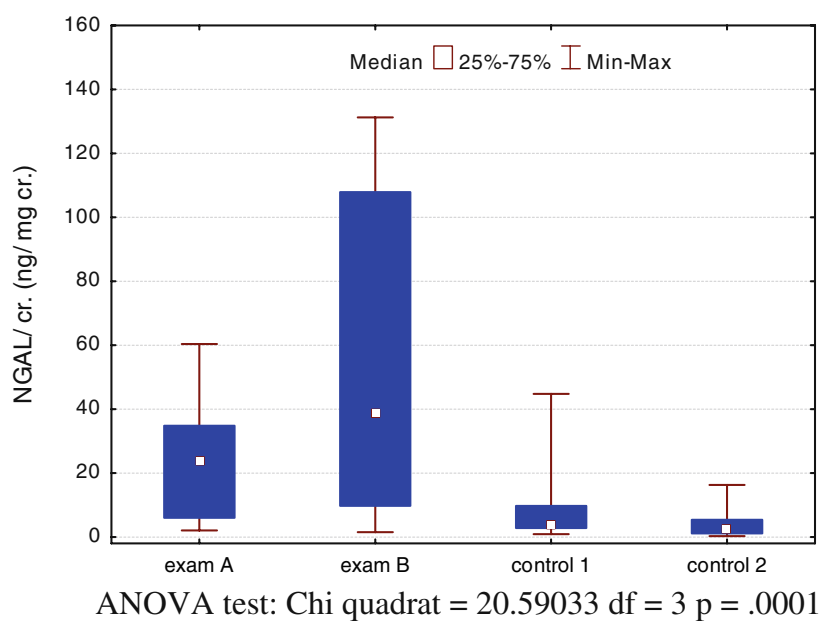

Fig. 1 uKIM-1/cr. (a) and uNGAL/cr. (b) excretion in patients with severe $\mathrm{HN}$ in exam A (before surgery) and exam B (urine from affected pelvis) and in patients with mild $\mathrm{HN}$ (control 1) and healthy children (control 2). uKIM-1 Urinary kidney injury molecule-1, $u N G A L$ urinary neutrophil gelatinase-associated lipocalin, $c r$. creatinine, ANOVA analysis of variance

Fig. 2 KIM-1/ cr. (a) and NGAL/ cr. (b) levels in patients who underwent pyeloplasty
A. uKIM/ cr.

B. uNGAL/ cr.
The correlation was even stronger between the uKIM-1/cr. levels from the affected pelvis and DRF $(r=-0.458, p<$ $0.01)$. A negative correlation was also found in control 1 group, but it was not statistically significant $(r=-0.213, p>$ 0.05 ). We did not find any significant correlation with serum creatinine level, age of patients, GFR, and initial AP diameter (length) of the pelvis.

The uNGAL/cr. level was found to be negatively correlated with DRF $(r=-0.422, p<0.05)$ and positively correlated with the $\mathrm{uKIM} / \mathrm{cr}$. level in children with severe HN $(r=0.377, p<0.05)$. No significant correlations with serum creatinine or GFR were found.

ROC analyses were performed to define the diagnostic profile of UKIM-1 and UNGAL in identifying children with an obstructive kidney condition ( $\mathrm{DRF}<40 \%$ ) among children with severe and mild HN (study group and control 1) and children with abnormal DRF $(<45 \%)$ among all examined children. In the first analysis in children with $\mathrm{HN}$, we found that both uKIM-1/cr. and uNGAL/cr. showed quite good diagnostic profile, describing an area under the curve (AUC) for uKIM-1/cr. of 0.8 [95\% confidence interval (CI) $0.687-0.914]$ with a best cut-off value of $0.462 \mathrm{ng} / \mathrm{mg}$ cr. (sensitivity $100 \%$, specificity $71.4 \%$ ) and an AUC for uNGAL/cr. of 0.814 (95\% CI $0.711-0.917)$ with a best cutoff value $2.158 \mathrm{ng} / \mathrm{mg}$ cr. (sensitivity $100 \%$, specificity $28.6 \%$ ). The difference in AUC between uKIM-1/cr. and uNGAL/cr. was $0.02(p>0.05)$. However, the results were analyzed in two different populations (younger children with severe $\mathrm{HN}$ and older control 1 patients).

In a subsequent analysis, we assessed the sensitivity and specificity of $\mathrm{uKIM}-1 / \mathrm{cr}$. and $\mathrm{uNGAL} / \mathrm{cr}$. as biomarkers of kidney injury $(\mathrm{DRF}<45 \%)$. In this analysis, the AUC for uKIM-1/ cr. was 0.779 (95\% CI 0.685-0.873) with a best cut-off value $0.997 \mathrm{ng} / \mathrm{mg}$ cr. (sensitivity $82.4 \%$, specificity $59.5 \%$ ), and the AUC for uNGAL/cr. was 0.868 (95\% CI 0.796-0.939) with a best cut-off value of $4.924 \mathrm{ng} / \mathrm{mg}$ cr. (sensitivity $82.4 \%$, specificity $75.7 \%$ ). Here, the AUC for uNGAL/cr. was higher; however the difference between the 
AUC for $\mathrm{uKIM}-1 / \mathrm{cr}$. and that for $\mathrm{uNGAL} / \mathrm{cr}$. was 0.08 and not statistically significant $(p>0.05)$.

ROC analyses for UKIM- 1 and UNGAL not corrected for urinary creatinine showed similar values.

\section{Discussion}

The future challenges of evaluating and treating patients with obstructive nephropathy relate to the indications for surgical intervention and finding prognostic indicators of renal progression. In clinical practice there are no clear indications for the timing of surgical intervention to prevent kidney injury. UPJO accounts for between 35 and $50 \%$ of all significant prenatally detected uropathies. However, the results of randomized trials suggest that only $19-29 \%$ of children with prenatally diagnosed UPJO require surgical intervention $[18,19]$. In this situation, although diuretic renography and US evaluation assist the clinician to establish the presence and degree of UPJO, there is an urgent need to look for potential biomarkers in patients' fluids, preferably urine, that would enable the early detection of obstructive nephropathy and deterioration in renal function.

We have evaluated the usefulness of KIM-1 and NGAL as biomarkers of obstructive nephropathy. To assess the utility of these biomarkers, we compared the magnitude of the alternation in their concentration in urine at different periods of time in children with confirmed $\mathrm{HN}$ with the levels in healthy children and in children with mild, nonobstructive HN. We also calculated the accuracy and sensitivity of each biomarker in children with $\mathrm{HN}$, according to the DRF of the affected kidney in a renal nuclide scan, using ROC analysis. To the best of our knowledge this study is the first published report that assesses the concentration of UKIM-1 and UNGAL in children with HN due to UPJO. Much progress has been made in the last decade in evaluating the mechanisms of obstructive nephropathy in UPJO, with the result that the following cellular mechanisms have been identified as major players: (1) tubular dilatation, phenotypic cellular transition, and cell death, (2) interstitial inflammation, and (3) glomerulotubular injury and progressive interstitial fibrosis [20].

In our study, the uKIM-1 levels were significantly elevated in patients who had developed an obstructed kidney but not yet undergone pyeloplasty. Three months after surgery, the concentration of UKIM-1 had decreased significantly, but did not reach the values found in children with dilated, but not obstructed kidney (control 1) $(p<$ 0.05 ). The uKIM-1 levels of patients from study group were significantly different from those of both control groups $(p<0.05)$. In contrast to the study of Ichimura et al. $[10,11]$, who did not detect uKIM-1 expression in healthy kidney, we detected low concentrations of uKIM-1 in the urine of our healthy controls.

What is the mechanism for increase of KIM-1 in patients with dilated and obstructed pelvis? KIM-1 is a protein that is localized at very high levels on the apical membrane of proximal tubule, which is the region where the tubules are more affected [21]. It has an ability to phagocytose apoptotic and necrotic cells in the tubules of the kidney and thus may be critical for remodeling after injury [22]. Chaturvedi et al. [13] confirmed that KIM-1 expression persists until the damaged cells have completely recovered, which might explain the fact that the uKIM-1 level was still elevated in our study patients 3 months after pyeloplasty. Interestingly, the difference in uKIM-1 concentration in voided urine before pyeloplasty and pelvic urine from the affected kidney was not statistically significant $(p>0.05)$. One possible explanation for this observation may be that intact contralateral kidney undergoes compensatory growth that is proportional to the duration of the obstruction [23]. In this situation, the source of the uKIM-1 in the voided urine may also be the contralateral kidney. We established a significant positive correlation between the levels of uKIM1 in voided urine and those in the affected pelvis, suggesting a valuable role for preoperative voided urine KIM-1 in predicting intrapelvic level.

The levels of UKIM-1 were negatively correlated with DRF in the radionuclide scan. The strong negative correlation between UKIM-1 levels in the pelvic urine and DRF of the affected kidney confirms the observation of Bonventre et al. [14] who found that uKIM-1 level is closely related to tissue KIM-1 and correlated with the severity of renal damage.

If we consider KIM-1 to be an early marker of tubular injury, we can hypothesize that UKIM-1 may express the degree of subclinical tubular impairment, thus representing an earlier measurable marker of its function. Recent data have confirmed KIM-1 expression in tubules of patients with various chronic proteinuric diseases, which was strongly associated with renal fibrosis and inflammation [15]. This has led to the proposal that KIM-1 can be considered as a non-invasive biomarker not only of the proximal tubules but also of chronic tubulointerstitial damage. This proposal is supported by data from our ROC analyses, which showed a good diagnostic profile for uKIM-1 in HN children (study and control 1 groups; AUC 0.8 ) and only a slightly worse profile for detecting kidney injury in $\mathrm{HN}$ children with DRF $<45 \%$ (AUC 0.779). The results may suggest that uKIM-1 may be useful in identifying the presence of obstruction in $\mathrm{HN}$ patients.

The second biomarker analyzed in this study was uNGAL, which has also been studied extensively in preclinical and clinical models and found to be a potential biomarker of number of pathologic conditions (e.g., AKI, 
diabetic nephropathy, IgA nephropathy, contrast nephropathy, breast cancer) and a useful tool in disease monitoring of other renal diseases (e.g., delayed graft function, lupus nephritis, IgA nephropathy and polycystic disease) $[9,24,25]$.

To the best of our best knowledge there are no published data on UNGAL levels in obstructive nephropathy. However in the mouse model of unilateral obstruction, NGAL protein synthesis was dramatically increased in the dilated thick ascending limb of Henle [8]. The authors found the increased NGAL levels in urine in the swollen pelvis of the ligated pelvis.

In our study, uNGAL levels were monitored in the same groups as was uKIM-1. uNGAL was detected in all of the healthy control children. In children with an obstructed kidney, the uNGAL concentration in voided urine (exam A) was tenfold higher and that in urine from the affected pelvis (exam B) was more than 16-fold higher than that in control 2 patients. Similar to the results for uKIM-1, uNGAL levels from the affected pelvis were not significantly higher than those in the voided urine $(p>0.05)$. Three months following the surgery, the UNGAL levels had decreased significantly $(p<0.05)$; however, they were still higher than those measured in control 2 patients $(p<0.05)$. Comparable to uKIM-1, uNGAL levels in control 1 children were lower than those in the study group, bu significantly higher than those in the healthy controls $(p<0.05)$. In addition, uNGAL levels correlated with uKIM-1 in children with HN.

A significant inverse correlation between uNGAL concentration and DRF was found in both the study and control 1 patients. Interestingly we did not find any relationship between NGAL concentration and serum creatinine concentration or GFR. As such, our results differ from those reported by Bolignano et al. [26], who confirmed significant correlations between uNGAL concentrations and serum creatinine concentration and GFR; however, their study was performed in patients with CKD. The lack of the correlation in our study might be explained by the fact that all of the children enrolled in our study had normal renal function.

The increased uNGAL levels observed in our study may express the degree of subclinical tubular impairment. Ding et al. [9] showed that NGAL protein is highly induced in proximal tubules in IgAN patients with tubulointerstitial injury and that this increase in this patient group may be due to its secretion from proximal tubular epithelial cells within damaged tubules to induce re-epithelialization. NGAL has also been found to be a regulator of epithelial morphogenesis in cultured kidney tubule cells [27]. Furthermore, Mishra et al. [28] suggested a possible role of NGAL in repairing damaged tubules. Devarajan [29] suggested that any urinary excretion of NGAL was likely to be present only when there was concomitant renal tubular injury that increased de novo NGAL synthesis and/ or precluded NGAL reabsorption. Increased uNGAL levels have been postulated to be an early predictor of severity of the microangiopathic disease and secondary to associated tubular damage in diarrhea-associated hemolytic uremic syndrome. Its role as a marker was also confirmed in kidney transplant recipients in terms of delayed graft function and acute rejection episodes. In one of our studies, we proposed that NGAL may be a non-invasive marker for the early detection of tubulointerstitial damage in cyclosporine A nephrotoxicity [30].

Data from the literature indicate that uNGAL measurement reflects local renal injury, which is the underlying rationale for it being proposed as a non-invasive marker in kidney injury due to $\mathrm{HN}$ in children. This suggestion is supported by the data from our ROC analyses, which showed a good diagnostic profile for uNGAL, possibly indicating that uNGAL may be of valuable in diagnosing significant obstruction.

The major limitation of this study is the number of patients, which is relatively small and does not allow us to draw an unequivocal conclusion. However, we were also unable to compare the examined parameters based on a histological diagnosis. It must also be taken into account that the increased levels of uKIM-1 and uNGAL may not only reflect renal tubular cell damage; there may also be extrarenal sources. Another point is that despite the high AUC values, the ROC analysis was performed in two different age populations (those with severe $\mathrm{HN}$ who were relatively younger and those with mild $\mathrm{HN}$ who were relatively older).

In conclusion, the results of our pilot study have clearly demonstrated that the children of our cohort with UPJO had increased urinary KIM-1 and NGAL levels and that these correlated negatively with DRF in the radionuclide scan. No less important, the results have a number of important diagnostic implications which have to be further validated in a larger population. The results suggest that UNGAL and uKIM-1 levels are associated with worsening obstruction. However, as this study is not a true prospective cohort study, we cannot draw the definitive conclusion that these markers are valuable in identifying obstruction. Future investigations are required to confirm a potential application of KIM-1 and NGAL as a useful biomarkers for such a diagnosis and as a parameters to prevent the development and progression of chronic kidney disease.

Acknowledgments The authors report no conflicts of interest in this work.

Open Access This article is distributed under the terms of the Creative Commons Attribution Noncommercial License which permits any noncommercial use, distribution, and reproduction in any medium, provided the original author(s) and source are credited. 


\section{References}

1. Smith JM, Stablein DM, Munoz R, Hebert D, McDonald RA (2007) Contributions of the transplant registry: The 2006 Annual Report of the North American Pediatric Renal Trials and Collaborative Studies (NAPRTCS). Pediatr Transplant 11:366-373

2. Chevalier RL, Thornhill BA, Forbes MS, Kiley SC (2010) Mechanisms of renal injury and progression of renal disease in congenital obstructive nephropathy. Pediatr Nephrol 25:687-697

3. Nickolas TL, Barasch J, Devarajan P (2008) Biomarkers in acute and chronic kidney disease. Curr Opin Nephrol Hypertens 17:127-132

4. Flower DR (1996) The lipocalin protein family: structure and function. Biochem J 318:1-14

5. Mishra J, Ma Q, Prada A, Mitsnefes M, Zahedi K, Yang J, Barasch J, Devarajan P (2003) Identification of neutrophil gelatinase-associated lipocalin as a novel early urinary biomarker for ischemic renal injury. J Am Soc Nephrol 14(10):2534-2543

6. Schmidt-Ott KM, Mori K, Li JY, Kalandadze A, Cohen DJ, Devarajan P, Barasch J (2007) Dual action of neutrophil gelatinase-associated lipocalin. J Am Soc Nephrol 18(2):407-413

7. Nickolas TL, O'Rourke MJ, Yang J, Sise ME, Canetta PA, Barasch N, Buchen C, Khan F, Mori K, Giglio J, Devarajan P, Barasch J (2008) Sensitivity and specificity of a single emergency department measurement of urinary neutrophil gelatinaseassociated lipocalin for diagnosing acute kidney injury. Ann Intern Med 148:810-819

8. Kuwabara T, Mori K, Mukoyama M, Kasahara M, Yokoi H, Saito Y, Yoshioka T, Ogawa Y, Imamaki H, Kusakabe T, Ebihara K, Omata M, Satoh N, Sugawara A, Barasch J, Nakao K (2009) Urinary neutrophil gelatinase-associated lipocalin levels reflect damage to glomeruli, proximal tubules, and distal nephrons. Kidney Int 75:285-294

9. Ding H, He Y, Li K, Yang J, Li X, Lu R, Gao W (2007) Urinary neutrophil gelatinase-associated lipocalin (NGAL) is an early biomarker for renal tubulointerstitial injury in $\operatorname{IgA}$ nephropathy. Clin Immunol 123:227-234

10. Ichimura T, Bonventre JV, Bailly V, Wei H, Hession CA, Cate RL, Sanicola M (1998) Kidney injury molecule-1 (KIM-1), a putative epithelial cell adhesion molecule containing a novel immunoglobulin domain, is up-regulated in renal cells after injury. J Biol Chem 273:4135-4142

11. Ichimura T, Hung CC, Yang SA, Stevens JL, Bonventre JV (2004) Kidney injury molecule-1: a tissue and urinary biomarker for nephrotoxicant-induced renal injury. Am J Physiol Renal Physiol 286:F552-F563

12. Vaidya VS, Ramirez V, Ichimura T, Bobadilla NA, Bonventre JV (2006) Urinary kidney injury molecule-1: a sensitive quantitative biomarker for early detection of kidney tubular injury. Am J Physiol Renal Physiol 290:F517-F529

13. Chaturvedi S, Farmer T, Kapke GF (2009) Assay validation for KIM-1: human urinary renal dysfunction biomarker. Int J Biol Sci $5: 128-134$

14. Bonventre JV (2009) Kidney injury molecule-1 (KIM-1): a urinary biomarker and much more. Nephrol Dial Transplant 24:3265-3268

15. van Timmeren MM, van den Heuvel MC, Bailly V, Bakker SJ, van Goor H, Stegeman CA (2007) Tubular kidney injury molecule-1 (KIM-1) in human renal disease. J Pathol 212:209-217
16. Fernbach SK, Maizels M, Conway JJ (1993) Ultrasound grading of hydronephrosis: introduction to the system used by the Society for Fetal Urology. Pediatr Radiol 23(6):478-480

17. Kajbafzadeh AM, Elmi A, Talab SS, Emami H, Esfahani SA, Saeedi P (2010) Urinary and serum carbohydrate antigen 19-9 as a biomarker in ureteropelvic junction obstruction in children. J Urol 183:2353-2360

18. Chertin B, Pollack A, Koulikov D, Rabinowitz R, Hain D, HadasHalpren I, Farkas A (2006) Conservative treatment of ureteropelvic junction obstruction in children with antenatal diagnosis of hydronephrosis: lessons learned after 16 years of follow-up. Eur Urol 49:734-738

19. Madden NP, Thomas DF, Gordon AC, Arthur RJ, Irving HC, Smith SE (1991) Antenatally detected pelviureteric junction obstruction. Is non-operation safe? Br J Urol 68:305-310

20. Chevalier RL (2006) Obstructive nephropathy: towards biomarker discovery and gene therapy. Nat Clin Pract Nephrol 2:157-168

21. Zhou Y, Vaidya VS, Brown RP, Zhang J, Rosenzweig BA, Thompson KL, Miller TJ, Bonventre JV, Goering PL (2008) Comparison of kidney injury molecule-1 and other nephrotoxicity biomarkers in urine and kidney following acute exposure to gentamicin, mercury, and chromium. Toxicol Sci 101:159-170

22. Huo W, Liu R, Zhang K, Nie J, Li Q, Jin F (2010) Kidney injury molecule-1: a novel kidney-specific injury molecule playing potential double-edged functions in kidney injury. J Nephrol. doi: $10.5301 /$ JN.2010.5800

23. Chevalier RL, Thornhill BA, Wolstenholme JT, Kim A (1999) Unilateral ureteral obstruction in early development alters renal growth: dependence on the duration of obstruction. J Urol 161:309-313

24. Brunner HI, Mueller M, Rutherford C, Passo MH, Witte D, Grom A, Mishra J, Devarajan P (2006) Urinary neutrophil gelatinase-associated lipocalin as a biomarker of nephritis in childhood-onset systemic lupus erythematosus. Arthritis Rheum 54:2577-2584

25. Bolignano D, Coppolino G, Campo S, Aloisi C, Nicocia G, Frisina N, Buemi M (2007) Neutrophil gelatinase-associated lipocalin in patients with autosomal-dominant polycystic kidney disease. Am J Nephrol 27:373-378

26. Bolignano D, Lacquaniti A, Coppolino G, Campo S, Arena A, Buemi M (2008) Neutrophil gelatinase-associated lipocalin reflects the severity of renal impairment in subjects affected by chronic kidney disease. Kidney Blood Press Res 31(4):255258

27. Gwira JA, Wei F, Ishibe S, Ueland JM, Barasch J, Cantley LG (2005) Expression of neutrophil gelatinase-associated lipocalin regulates epithelial morphogenesis in vitro. J Biol Chem 280:7875-7882

28. Mishra J, Mori K, Ma Q, Kelly C, Yang J, Mitsnefes M, Barasch J, Devarajan P (2004) Amelioration of ischemic acute renal injury by neutrophil gelatinase-associated lipocalin. J Am Soc Nephrol 15:3073-3082

29. Devarajan P (2008) Emerging urinary biomarkers in the diagnosis of acute kidney injury. Expert Opin Med Diagn 2:387-398

30. Wasilewska A, Zoch-Zwierz W, Taranta-Janusz K, MichalukSkutnik J (2010) Neutrophil gelatinase-associated lipocalin (NGAL): a new marker of cyclosporine nephrotoxicity? Pediatr Nephrol 25:889-897 